Pacific Journal of Mathematics

MEIER TYPE THEOREMS FOR GENERAL BOUNDARY
APPROACH AND $\sigma$-POROUS EXCEPTIONAL SETS 


\title{
MEIER TYPE THEOREMS FOR GENERAL BOUNDARY APPROACH AND $\sigma$-POROUS EXCEPTIONAL SETS
}

\author{
D. C. RUNG
}

In this paper we are concerned with determining under what conditions equality is obtained between two different cluster sets of a function $f$ at a point on the boundary of its domain. Specifically for functions defined in the unit disc $D$ in the complex plane taking values in the extended plane we show that the generalized angle cluster set equals the generalized outer angular cluster set at all points of the boundary of $D$ except possibly for a $\sigma$-porous set. The definition of both generalized cluster sets includes the usual Stolz angle definition but this result generalizes the known results. In addition the proof is shorter than proofs of less general results.

If $f$ is required to be meromorphic in $D$ then an application of the principal result gives a decomposition of the boundary of $D$ into a set of generalized normal points of $f$, a set of generalized Picard points of $f$, and a $\sigma$-porous set. The third result gives a different decomposition into generalized Plessner points; generalized pre-Meier points and a $\sigma$-porous set. Again these results generalize known results.

The notion of porosity was introduced in 1967 by E. P. Dolženko. While he defined porous sets in higher dimensions as well we shall limit our considerations to $C:|z|=1$. Porous sets have zero Lebesgue measure and are of first Baire category and in addition isolate properties essential for certain cluster set considerations. In turn this allows generalizations of results of Meier [10] and others. In a series of papers Yoshida [14-20] extended results of Meier and others by using the notion of porosity. Yoshida's fundamental lemma [15, Lemma 1] has a very complicated statement and proof. In this paper we expand this lemma; offer a succinct proof which hopefully illuminates better the character of the results; and apply it to obtain generalizations of some of Yoshida's results as well as those of Dragosh ([6], [7]), Colwell [4] and Yanigahara [13].

2. Definitions and notation. Let $D$ be the open unit disc and $P \subseteq C$. For each $e^{i \theta} \in C$, let $\gamma(\theta, \varepsilon, P)$ be the length of the largest subarc of the $\operatorname{arc}\left(e^{i(\theta-\varepsilon)}, e^{i(\theta+\varepsilon)}\right)$ which does not meet $P$. If no such arc exists define $\gamma(\theta, \varepsilon, P)=0$. According to Dolženko [5], $P$ is porous at $e^{i \theta}$ if 


$$
\varlimsup_{\varepsilon \rightarrow 0} \frac{\gamma(\theta, \varepsilon, P)}{\varepsilon}>0
$$

Definition 1. A subset $P \subseteq C$ is a porous set if it is porous at each $p \in P ; P$ is $\sigma$-porous if it is the finite or countable union of porous sets.

Condition (2.0) implies that the density of $P$ at $e^{i \theta}$ is not unity and so a porous set must have zero Lebesgue measure as does a $\sigma$ porous set. Because a porous set is nowhere dense, a $\sigma$-porous set is also of first Baire category. Thus porosity contains both the metric and topological features found in Meier-type theorems. It is known [3, p. 75] there exists a residual set on $C$ with zero Lebesgue measure and so zero Lebesgue measure does not imply $\sigma$-porosity. The set of rationals is an example of a set of first category and measure 0 which is not porous. The existence of a set which is of first category and measure 0 but not $\sigma$-porous was asserted by Dolženko [5, pg. 3] and was constructed by Zajiček [21, Prop. 5.2].

Zero capacity and $\sigma$-porous sets do not have any obvious connections. If $E$ is the Cantor middle thirds set on $C$ and $e^{i \theta} \in E$, it is easy to see that $\gamma\left(\theta, 1 / 6^{n}, E\right) \geqq\left(1 / 6^{n}\right)(2 / 3), n=1,2, \cdots$, and so $E$ is porous. On the other hand $E$ is known to have positive capacity. To complete the analysis we construct a residual set $K$ (thus $K$ is not $\sigma$-porous) which has zero capacity. It will be the intersection of complements of Cantor-type set. For convenience we construct the set on $[0,1]$. Begin by letting $H_{1}$ be the closed interval of length $1 / 4$ symmetric about $1 / 2$; then let $\left\{F_{i}^{(2)}\right\}, i=1,2$, be the closed intervals symmetric about $1 / 2^{2}$ and $3 / 2^{2}$ respectively, with each interval of equal length and chosen so that, setting $H_{2}=F_{1}^{(2)} \cup F_{2}^{(2)} \cup H_{1}$,

$$
\operatorname{cap} H_{2} \leqq \operatorname{cap} H_{1}+\frac{1}{2^{2}}
$$

This is clearly possible. Proceed inductively defining $H_{n}$ by adjoining to $H_{n-1}, 2^{n-1}$ closed intervals of equal length symmetric about the points $i / 2^{n}, i=1,3, \cdots, 2^{n}-1$ and chosen so that

$$
\operatorname{cap} H_{n} \leqq \operatorname{cap} H_{n-1}+\frac{1}{2^{n}}
$$

The $H_{n}$ 's are closed sets with $H_{n-1} \cong H_{n}$ and thus setting $K_{1}=$ $\bigcup_{n=1}^{\infty} H_{n}$,

$$
\operatorname{cap} K_{1}=\lim _{n \rightarrow \infty} \operatorname{cap} H_{n} \leqq \lim _{n \rightarrow \infty}\left[\left(\operatorname{cap} H_{1}\right)+\sum_{k=2}^{n} \frac{1}{2^{k}}\right]=\frac{1}{16}+\frac{1}{2}=\frac{9}{16} .
$$

Let $K_{2}$ be the set obtained by the same method as used for $K_{1}$ except 
that we begin by selecting the initial interval about $1 / 2$ with capacity $1 / 32$, and all subsequent closed intervals symmetric about the same midpoints but chosen so that the new $H_{n}$ 's satisfy

$$
\operatorname{cap} H_{n} \leqq \operatorname{cap} H_{n-1}+\frac{1}{2^{n+1}} \text {. }
$$

Thus $K_{2} \subseteq K_{1}$, and cap $K_{2} \leqq 9 / 2^{3}$. Defining $K_{n}, n>2$, in the same fashion and setting $K=\bigcap_{n=1}^{\infty} K_{n}$, we have

$$
\operatorname{cap} K=0 \text {. }
$$

Because each $K_{n}$ is a residual set so also is $K$. (I wish to thank Professor W. Schneider for suggesting this construction.)

For future use we note that if $P$ is not $\sigma$-porous then the subset $P^{*}$ of $P$ of all points at which $P$ is not porous is uncountable. Otherwise since $P-P^{*}$ is porous - as is easily seen-and a singleton set is obviously porous we would have that $P$ is $\sigma$-porous.

Definition 2. If a property $\mathscr{P}$ holds for all points of $E \subseteq C$ except possibly on a $\sigma$-porous subset of $E$, we say that $\mathscr{P}$ holds strongly on $E$, or that strongly on $E$ points satisfy property $\mathscr{P}$.

Substs of $C$ which are not $\sigma$-porous have the happy property of exerting strong collective influence on the boundary behavior of functions $f$ at certain points of $C$. This is best illustrated by Theorem 3, although out first lemma indicates the basic connection between porosity and boundary behavior. This lemma was proved in the angular case by Dolženko [5, Eng. translation, pg. 5], and in a more general setting by Yoshida [15, Lemma 1], although Dragosh [6, Lemmata 4, 5] proved a horocyclic version using metric density instead of porosity. We present it in a still more general setting and a result of Satyanarayana and Weiss [12, Theorem 5] both simplifies the proof and extends Yoshida's result. (We thank Professor L. Garbanati for this last reference.)

We wish to define approach to a point of $C$ inside a reasonably nice subdomain of $D$ and so let $h(\varphi)$ be a real valued function defined in some open interval $I_{h}$ on the real line symmetric about 0 . On $I_{h}$ we require that

$$
\begin{aligned}
& \text { (i ) } h \text { be continuous; } \\
& \text { (ii) } h(\varphi)=h(-\phi) \text {; }
\end{aligned}
$$$$
\text { (iii) } h(0)=0, h(1)=1, h\left(\varphi_{1}\right)<h\left(\varphi_{2}\right)<1,0 \leqq \varphi_{1}<\varphi_{2} \text {. }
$$

Call such an $h$ an approach function. In addition if $h^{\prime \prime}(\varphi)$ exists for $\varphi \in I_{h}, \varphi \neq 0$, and is strictly positive, then $h$ is said to be a convex 
approach function. An approach function $h$ defines a boundary curve at $e^{i \theta} \in C$ as follows. Let $\phi-\theta \in I_{h}$ and set

$$
z(\varphi ; \theta, h)=(1-h(\varphi-\theta)) e^{i \varphi} \text {. }
$$

Then $z(\varphi ; \theta, h)$ is a symmetric curve with respect to the radius at $e^{i \theta}$ and determines a boundary domain at $e^{i \theta}$, according to the following

$$
\Omega(\theta, h)=\left\{\mathrm{re}^{i \varphi} \mid r \leqq 1-h(\varphi-\theta)\right\} .
$$

Note that when $\left|I_{h}\right|<\pi, \Omega$ is convex if $h$ is. For $0 \leqq r<1$, a truncated boundary domain is

$$
\Omega_{r}(\theta, h)=\Omega(\theta, h) \cap\{|z| \geqq r\} .
$$

Henceforth if $A \leqq D, A_{r}$ will mean $A \cap\{|z| \geqq r\}$. If $h(\varphi)=\phi^{q+1}$, $q \geqq 0$, the case $q=0$, defines the usual nontangential approach; for $q=1$, we have horocyclic approach; and for arbitrary $q \geqq 0$, the situation considered by Yoshida. For $q>0$ it is necessary to define right and left $h$-angles at $e^{i \theta}$. To this end for $c>0$ put $h^{c}(\varphi)=h(\varphi / c)$, and for $0<a<b$, define the right angle

$$
R \mathscr{A}(\theta, a, b, h)=\left\{\mathrm{re}^{i \varphi} \mid 1-h^{a}(\varphi-\theta)<r<1-h^{b}(\varphi-\theta) ; \varphi \geqq \theta\right\},
$$

while a left $h$-angle $L \mathscr{A}(\theta, a, b, h)$ is defined as in (2.5) except $\rho \geqq \theta$ is replaced by $\varphi \leqq \theta$. A reduced right $h$-angle $R \alpha(\theta, a, b, h)$ (or reduced left $h$-angle) is defined by replacing $h^{a}$ and $h^{b}$ in (2.5) by $h / a$ and $h / b$ respectively. Any choice of $0<a<b$ defines a right and left $h$-angle and we omit any of the parameters in the symbol if we can safely ignore them.

The reduced $h$-angle $\alpha$ is so called because for a convex approach function $h$, and any $0<a<b$,

$$
R \alpha\left(\theta, \frac{2 a}{a+b}, \frac{2 b}{a+b}, h^{(a+b) / 2}\right) \cong R \mathscr{A}(\theta, a, b, h) ;
$$

with left $h$-angles also satisfying (2.6). To see this we combine the definition of angles in (2.5) and the fact that for convex approach functions $h^{\prime}$ is increasing for $\phi \neq 0$ to argue via the generalized mean value theorem that

$$
\frac{\frac{a+b}{2 a} h\left(\frac{(\varphi-\theta) 2}{a+b}\right)}{h\left(\frac{\varphi-\theta}{a}\right)}=\frac{\left(\frac{a+b}{2 a}\right)\left(\frac{2}{a+b}\right) h^{\prime}\left(\frac{(\overline{\varphi-\theta}) 2}{a+b}\right)}{\frac{1}{a} h^{\prime}\left(\frac{\overline{\varphi-\theta}}{a}\right)}>1 .
$$

A similar result holds for the other pair of boundary curves. Because $h^{(a+b) / 2}$ is a convex approach function the result of Satyanaraya and 
Weiss [12, Theorem 5] implies that the hyperbolic Hausdorff distance at $e^{i \theta}$ between the boundary curves of the reduced $h$-angle in (2.6) is asymptotic to $1 / 2 \log (1+2|a-b| / 1-2|a-b|)$ as $|z| \rightarrow 1$, and so not only does a right $h$-angle defined by a convex $h$ contain a reduced $h$-angle but one of a positive hyperbolic width.

Let $\rho(\alpha, b)$ denote the hyperbolic distance in $D$ and $N(a, s)=$ $\{b \in D \mid \rho(a, b)<s\}$. The above result reinterpreted says that a (reduced) $h$-angle at $e^{i \theta}$-provided $h$ is convex-contains (close to $e^{i \theta}$ ) discs of a fixed hyperbolic radius. Finally note that if $h(\theta)=k \theta^{q+1}$, $q \geqq 0, k>0$, then $h$-angles and reduced $h$-angles are equivalent.

3. Fundamental lemma. The lemma first is given in a settheoretic fashion using approach functions, and then in a hyperbolic geometric setting using convex approach functions.

Lemma 1 (Spotlight Lemma). Let $P \subseteq C$, such that $P$ is not porous at $e^{i \theta}$. Given an apprgach function $h$ and positive numbers $a<b, r^{\prime}$, c, there is a value $r>0$, such that

$$
\Omega_{r}\left(\theta ; h^{c}\right) \subseteq \bigcup_{e^{i \varphi} \in P} R \mathscr{A}_{r^{\prime}}(\varphi, a, b, h) ;
$$

and the same result holds for left h-angles.

REMARK. The right $h$-angles are congruent under rotation and can be thought of as beams of light with a positive width emanating from each point of $P$. The beams are hyperbolically wide if $h$ is convex. The lemma says that if all the lights are turned on there is an $r>0$ such that the set $\Omega_{r}\left(\theta, h^{c}\right)$ is fully illuminated.

Proof. It is easy to show that it is sufficient to prove the lemma for the case $c=1$, and $\theta=0$. We first consider the right angle situation. Suppose the lemma is false. Then for some choice of $0<a<b$ and $r^{\prime}>0$, there is a sequence $\left\{z_{n}\right\}$ tending to 1 inside $\Omega(0, h)$ such that

$$
z_{n} \notin \bigcup_{e^{i \theta} \in P} R \mathscr{A}_{r^{\prime}}(\theta, a, b, h),
$$

that is, the $z_{n}$ lie in the umbra of all right $h$-angle lights at $P$. Let $z\left(\varphi ; \theta_{n}^{a}, h^{a}\right)$ and $z\left(\varphi ; \theta_{n}^{b}, h^{b}\right)$ be the unique $h$-angle boundary curves through $z_{n}$. See Figure 1 for a description of these and other pertinent sets. Assume first that $\theta_{n}^{b}<\theta_{n}^{a}<0$. The arc $\left(e^{i \theta_{n}^{h}}, e^{i \theta_{n}^{a}}\right)$ then lies in the complement of $P$. We will show that the ratio

$$
\frac{\theta_{n}^{b}-\theta_{n}^{a}}{\theta_{n}^{b}}
$$




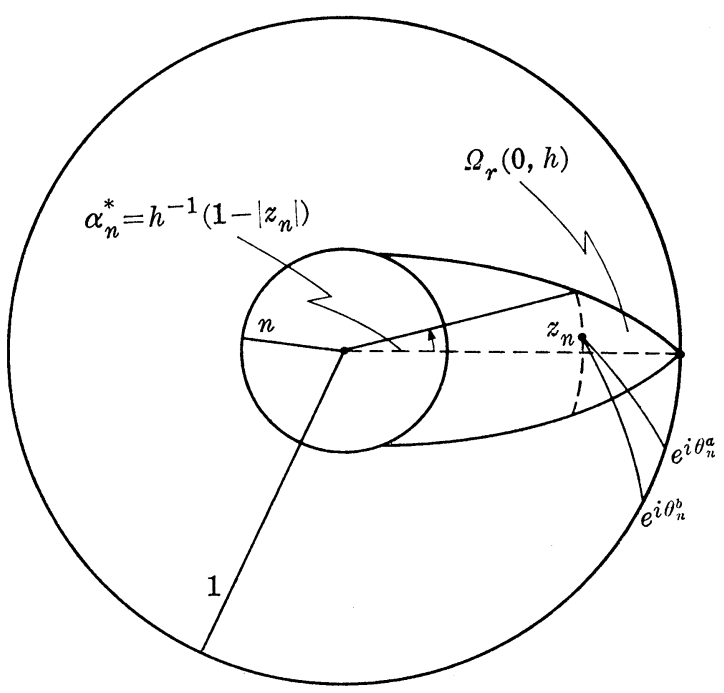

FIGURE 1

is bounded away from zero which contradicts the porosity hypothesis. The two curves $z\left(\varphi ; \theta_{n}^{a}, h^{a}\right)$ and $z\left(\varphi ; \theta_{n}^{b}, h^{b}\right)$ were chosen to meet at $z_{n}=\left|z_{n}\right| e^{i \varphi_{n}}$. Thus

$$
\left|z_{n}\right|=1-h\left(\frac{\varphi_{n}-\theta_{n}^{a}}{a}\right)=1-h\left(\frac{\varphi_{n}-\theta_{n}^{b}}{b}\right) .
$$

On the boundaries of right $h$-angles $h^{-1}$ exists and is positive. From (3.3)

$$
\frac{\varphi_{n}-\theta_{n}^{a}}{a}=h^{-1}\left(1-\left|z_{n}\right|\right)=\frac{\varphi_{n}-\theta_{n}^{b}}{b} .
$$

Since $z_{n} \in \Omega(0, h)$ we have that $\left|z_{n}\right|<1-h\left(\varphi_{n}\right)$ and after applying $h^{-1}$ to this inequality

$$
\frac{\left|\varphi_{n}\right|}{h^{-1}\left(1-\left|z_{n}\right|\right)}<1
$$

Using (3.4) and (3.5), the ratio in (3.2) equals

$$
\frac{b-a}{b-\frac{\varphi_{n}}{h^{-1}\left(1-\left|z_{n}\right|\right)}}>\frac{b-a}{b+1}>0 \text {. }
$$

Thus a contradiction is reached. If $0 \leqq \theta_{n}^{b}<\theta_{n}^{a}$ a lower bound for $\left(\theta_{n}^{a}-\theta_{n}^{b}\right) / \theta_{n}^{a}$ is found to be $b-a / a+1$, while if $\theta_{n}^{b}<0<\theta_{n}^{a}, P$ is porous at 1 by definition. The proof for left angles is done by an entirely similar analysis. 
Lemma 2. Let $P \subseteq C$, and suppose $P$ is not porous at $e^{i \theta}$. Let $h$ be a convex approach function and $0<a<b, n^{\prime}>0$ and $c>0$ be given. Then there exists $r>0, s>0$, such that for each sequence $\left\{z_{n}\right\}$ tending to $e^{i \theta}$ in $\Omega_{r}\left(\theta, h^{c}\right)$, there is a corresponding sequence $\left\{e^{i \varphi_{n}}\right\}$ in $P$, with the property that

$$
N\left(z_{n}, s\right) \subseteq R \mathscr{A}_{r^{\prime}}\left(\varphi_{n}, a, b, h\right), \quad \text { all } n .
$$

A corresponding statement holds for left h-angles as well.

Proof. Choose $0<a<a^{\prime}<b^{\prime}<b$. Then by Lemma 1 the collection of rays $R \mathscr{A}_{r^{\prime}}\left(\varphi, a^{\prime}, b^{\prime}, h\right), e^{i \varphi} \in P$, cover $\Omega_{r}\left(\theta, h^{c}\right)$ for some $r>0$. Select a point $e^{i \theta_{n}} \in P$ for which $z_{n} \in R \mathscr{A}_{r^{\prime}}\left(\varphi_{n}, a^{\prime}, b^{\prime}, h\right)$. Of course $z_{n}$ is also in the larger ray $R \mathscr{A}_{r^{\prime}}\left(\varphi_{n}, a, b, h\right)$-larger by a fixed hyperbolic distance according to the remarks following (2.7) and the observation that each of the two sets between the larger and smaller rays is itself a ray. Consequently $R \mathscr{A}_{r^{\prime}}\left(\varphi_{n}, a, b, h\right)$ contains $N\left(z_{n}, s\right)$ from somc $s>0$, and all $n$, and the right $h$-angle statement of the lemma is proved. A similar argment validates the left angle case.

If $h_{1}$ and $h_{2}$ are two approach functions with $h_{2}<h_{1}$, and if some collection of $h_{2}$ rays cover $\Omega_{r}\left(\theta, h_{2}\right)$, the same collection also cover $\Omega_{r}\left(\theta, h_{1}\right)$. For example if a collection of horocyclic rays cover an oricyclic domain they also cover any angular domain.

4. Consequences of the spotlight lemma. The first consequence of Lemma 1 is a statement about cluster sets of arbitrary functions and generalizes the results of Dragosh [6, Lemma 6], Doležnko [5, Theorem 1], Yoshida [15, Theorem 2] and Yanagihara [13]. If $E \subseteq D$ such that $e^{i \theta} \in \bar{E} \cap C$, then the cluster set of a function $f$ along $E$ at $e^{i \theta}$ will be denoted by $C(f, \theta, E)$, with again any parameter omitted if safely done. And let $W$ be the extended plane.

THEOREM 1. Let $f$ be defined in $D$ taking values in $W$, and $h$ a given approach function. Then strongly on $C$, for any choice of $0<a<b$, and $c>0$,

$$
C(f, \mathscr{A}(\theta, a, b, h))=C\left(f, \Omega\left(\theta, h^{c}\right)\right) .
$$

Proof. There is no loss of generality in assuming all angles are right $h$-angles. Further by a simple argument it is easy to show that we need prove the result only in the case $a, b$, and $c$ are rational with $b<c$, and so we suppose the result is false in this case. Then for some rational numbers $0<a<b<c$ there exists a non- $\sigma$-porous set $E \subseteq C$ such that for each $e^{i \theta} \in E$ the set on the left in (4.0) is a 
proper subset of the set on the right. Select any countably dense subset $P$ of $W$ and let $R$ be the positive rationals. Then $e^{i \theta} \in E$ if and only if for some choice of $p_{0} \in P$ and $r_{0} \in R$ the closed disk $D\left(p_{0}, r_{0}\right)$ with center $p_{0}$ and radius $r_{0}$ is entirely contained in the complement of $f\left(\mathscr{A}_{r^{\prime}}(\theta, a, b, h)\right)$ for some rational $r^{\prime} \in R$, and such that $D\left(p_{0}, r_{0} / 2\right) \cap C\left(f, \Omega\left(\theta, h^{r_{1}}\right)\right) \neq \varnothing$. The set of all $e^{i \theta} \in E$ which satisfies the above situation relative to the parameters $\left(a, b, c, r^{\prime}, p_{0}, r_{0}\right)$ we denote by $E\left(a, b, c, r^{\prime}, p_{0}, r_{0}\right)$. Thus $E$ is the countable union of all such $E\left(a, b, c, r^{\prime}, p_{0}, r_{0}\right)$ :

$$
E=U E\left(a, b, c, r^{\prime}, p_{0}, r_{0}\right) .
$$

Then at least one set in the union (4.1) is not porous. Let $E\left(a, b, c, r^{\prime}, p_{0}, r_{0}\right)=\widetilde{E}$ be such a nonporous set and select a point $e^{i \theta} \in \widetilde{E}$ at which $\widetilde{E}$ is not porous. From Lemma 1 there is some value $r>0$ for which

$$
\bigcup_{e^{i \varphi} \in \widetilde{E}} \mathscr{A}_{r^{\prime}}(\varphi, a, b, h) \supseteqq \Omega_{r}\left(\theta, h^{c}\right) .
$$

From our definition of $\widetilde{E}$ we know that if $e^{i \varphi} \in \widetilde{E}$ then $f\left(\mathscr{A}_{r^{\prime}}(\varphi, a, b, h)\right)$ omits the closed disk with center $p_{0}$ and radius $r_{0}$ and so (4.2) implies that $f\left(\Omega_{r}\left(\theta, h^{c}\right)\right)$ also omits this same closed disk. But then $C\left(f, \Omega\left(\theta, h^{c}\right)\right)$ does not meet $D\left(p_{0}, r_{0} / 2\right)$ contrary to the fact that $e^{i \theta} \in \widetilde{E}$. Indeed $E$ is $\sigma$-porous.

To state some immediate consequences of the lemma in familiar form we need some more definitions. Let $h$ be an approach function and $f$ a function in $D$ with values in $W$. A point $e^{i \theta}$ is called an $h$-Plessner (h-Fatou) point of $f$ if $C(f, \mathscr{A}(\theta, h))=W(C(f, \mathscr{A}(\theta, h))=$ $\left.\left\{w_{0}\right\}\right)$, for all $h$-angles at $e^{i \theta}$. For $h(\theta)=\theta$ we obtain the standard Plessner (Fatou) point. The $h$-Fatou value $w_{0}$ is to be independent of the particular $h$-angle used. If we use reduced $h$-angles in place of standard $h$-angles in the above we say $e^{i \theta}$ is a reduced $h$-Plessner or reduced h-Fatou point.

Henceforth we shall always assume a sequence $\left\{h_{n}\right\}$ of approach functions has the two additional properties that $h_{n} \geqq h_{n+1}$, and $h_{1}(\theta)=\theta$. If $e^{i \theta}$ is an $h_{n}$-Plessner $\left(h_{n}\right.$-Fatou) point of $f$ for every $h_{n} \in \mathscr{H}$, we say $e^{i \theta}$ is an $\mathscr{H}$-Plessner ( $\mathscr{C}$-Fatou) point of $f$. In the Fatou case we do not assume that the various $h_{n}$-Fatou values are equal. The sequence of polynomial approach functions $h_{n}(\theta)=\theta^{n}$, $n=1,2, \cdots$, we call $\mathscr{P}$. Finally let $K_{h}(f)$ be the set of all points $e^{i \theta}$ at which the cluster sets of $f$ along any two $h$-angles at $e^{i \theta}$ are equal, and put $K_{\mathscr{C}}(f)=\bigcap_{h \in \mathscr{O}} K_{\mathscr{C}}(f)$. Because a countable union of $\sigma$-porous sets is again $\sigma$-porous Theorem 1 can be rephrased as 
THEOREM 1'. Let $\mathscr{H}=\left\{h_{n}\right\}$ be a sequence of approach functions and $f$ a function in $D$. Then strongly (i) Plessner points of $f$ are $\mathscr{H}$-Plessner points of $f$; (ii) for any fixed $n$ the $h_{n}$-Fatou points of $f$ are also $h_{k}$-Fatou points for any $k \leqq n$ (with the same $h_{n}$-Fatou value); (iii) $K_{*}(f)$ is all of $C$.

To arrive at Meier-type theorems we need to limit ourselves to meromorphic functions and state a few more definitions. For $e^{i \theta} \in C$, let $\Lambda(f, \theta, h)$ be the set of all values $w \in W$ such that $w$ is assumed infinitely often by $f$ in each (right or left) $h$-angle at $e^{i \theta}, h$ an approach function. If we use only right (left) $h$-angles in the above we write $R \Lambda(f, \theta, h)(L \Lambda(f, \theta, h))$. If $W-\Lambda(f, \theta, h)$ contains at most two values then $e^{i \theta}$ is called an $h$-Picard point of $f$. Let $\chi$ be the chordal distance on $W$. A point $e^{i \theta}$ is an h-normal point of $f$ provided for each $c>0$, and for any two sequences $\left\{z_{n}\right\}$ and $\left\{z_{n}^{\prime}\right\}$ with $z_{n} \in \Omega\left(\theta, h^{c}\right)$ all $n$, the property $\rho\left(z_{n}, z_{n}^{\prime}\right) \rightarrow 0, n \rightarrow \infty$ implies $\chi\left(f\left(z_{n}\right), f\left(z_{n}^{\prime}\right)\right) \rightarrow 0, n \rightarrow \infty$. (In the angular case this is a definition of J. M. Anderson [1, p. 103].) If $\mathscr{H}=\left\{h_{n}\right\}$ is a sequence of approach functions and $e^{i \theta}$ is an $h_{n}$-Picard $\left(h_{n}\right.$-normal) point of $f$ for each $h_{n} \in \mathscr{H}$ then $e^{i \theta}$ is said to be an $\mathscr{H}$-Picard ( $\mathscr{C}$-normal) point of $f$.

THEOREM 2. Let $f$ be meromorphic in $D$ and $h$ a convex approach function. Let $Q \subseteq C$ be a set of non-h-Picard points of $f$. If $Q$ is not $\sigma$-porous there exists a nondenumerable set $Q^{*} \cong Q$ of $h$-normal points of $f$.

Proof. In the fashion of Theorem 1 - and following Meier [10]we write $Q$ as a countable union of fairly nice sets. Let $R$ be the positive rationals. In addition let $\mathscr{T}$ be the (countable) collection of triples $\left\{D_{1}, D_{2}, D_{3}\right\}$, where each $D_{i}, i=1,2,3$, is a closed disc in $W$ with rational center and radius and the triple of sets are pairwise disjoint. Thus there exists a $d_{T}>0$ such that if $T=$ $\left(D_{1}, D_{2}, D_{3}\right)$, then $\chi\left(D_{i}, D_{j}\right) \geqq d_{T}, i \neq j$. Distinguish the set of all points $e^{i \varphi} \in Q$ at which $f$ omits some three values $w_{i}^{(\varphi)}, i=1,2,3$, (all distinct), respectively in the three truncated $h$-angles at $e^{i \theta}$, $\mathscr{A}_{r_{1 i}}\left(\varphi, r_{2 i}, r_{3 i}, h\right)$ each $r_{h i} \in R$ and $w_{i}^{(\varphi)} \in D_{i}, i=1,2,3$, for some $T=$ $\left(D_{1}, D_{2}, D_{3}\right) \in \mathscr{T}$. Thus

$$
\chi\left(w_{i}^{(\varphi)}, w_{j}^{(\varphi)}\right) \geqq d_{T}, \quad i \neq j ; \quad i, j=1,2,3 .
$$

The three $h$-angles associated with the triad $\left(w_{1}^{(\varphi)}, w_{2}^{(\varphi)}, w_{3}^{(\varphi)}\right)$ are one of eight possible combinations of $h$-angles according as to whether they are left or right angles. We use $\kappa=1,2, \cdots, 8$ to designate the combinations. 
Label the set of all $e^{i \varphi} \in Q$ satisfying the above conditions as $Q\left(\left\{r_{k i}\right\}_{i, i=1}^{3}, T, \kappa\right)$. Then $Q$ is the countable union of all such sets. Consequently at least one set in the union, say $\widetilde{Q}$ is not porous, and let $Q^{*}$ be the nondenumerable subset of $\widetilde{Q}$ at which $\widetilde{Q}$ is not porous. To show any $e^{i \theta} \in Q^{*}$ is a $h$-normal point of $f$ fix a domain of the form $\Omega\left(\theta, h^{c}\right)$ and consider two sequences $\left\{z_{n}\right\}$, $\left\{z_{n}^{\prime}\right\}$, with $z_{n} \in \Omega\left(\theta, h^{c}\right)$ and $\rho\left(z_{n}, z_{n}^{\prime}\right) \rightarrow 0, n \rightarrow \infty$. Notice that for each value $i=1,2,3$, the corresponding collection of $h$-angles emanating from points of $\widetilde{Q}$ is composed of all right or left $h$-angles and so by Lemma 2 where are three sequences in $\widetilde{Q}, e^{\varphi_{n}^{(i)}}, i=1,2,3$, such that for all $n>N_{i}$, and some $\rho_{i}>0$

$$
N\left(z_{n}, \rho_{i}\right) \leqq \mathscr{A}_{r_{1 i}}\left(\varphi_{n}^{(i)}, r_{2 i}, r_{3 i}, h\right), \quad i=1,2,3 .
$$

Thus $f$ omits all three values $\left\{w_{i}^{\varphi}{ }^{(i)}\right\}, i=1,2,3$, in $N\left(z_{n}, \rho_{0}\right), \rho_{0}=$ $\min \rho_{i}, \quad i=1,2,3$, for $n$ sufficiently large, and the triad of points $\left\{w_{i}^{\varphi^{(i)}}\right\}$ satisfies (4.3) for fixed $d_{T}$. According to Gauthier [8, p. 281], this implies that $\chi\left(f\left(z_{n}\right), f\left(z_{n}^{\prime}\right)\right) \rightarrow 0, n \rightarrow \infty$, that is, $e^{i \theta}$ is an $h$-normal point. The result of Gauthier cited here involves the $\rho$-sequences of Lange [9].

This result which generalizes Dragosh [7, Theorem 9] and Yoshida [17, Theorem 4] can be phrased as a strong decomposition of $C$.

THEOREM 2'. Let $f$ be meromorphic in $D$ and $\mathscr{H}$ a sequence of convex approach functions. Then strongly on $C$ points are either $\mathscr{H}$-Picard points of $f$ or $\mathscr{C}$-normal points of $f$.

Proof. For any $h \in \mathscr{H}$ the set of points of $C$ which are neither $h$-Picard points nor $h$-normal points of $f$ must be $\sigma$-porous else by Theorem 2 that set contains $h$-normal points.

The theorems of Meier, Yoshida, and to some extent Dragosh previously mentioned arise by exploiting the behavior of $f$ at an $h$-normal point. To generalize and strengthen these theorems a few more standard cluster sets need to be defined. Because we rely on the usual normal function arguments we sometimes have to use both convex approach functions and reduced angles in order to insure that these angles have a positive hyperbolic distance from boundary to boundary. Again we mention that for approach functions of the form $h(\theta)=\theta^{q+1}, q \geqq 0, h$-angles and reduced $h$-angles are equivalent.

Let $h$ be a given approach function and $f$ a complex function in $D$. For $e^{i \theta} \in C$, let

$$
\pi(f, \theta, h)=\bigcap_{\gamma} C(f, \theta, \gamma),
$$


where the intersection is taken over all curves $\gamma$ which tend to $e^{i \theta}$ and which have the property that each $\gamma$ is contained in its own reduced $h$-angle at $e^{i \theta}$. A curve tending to $e^{i \theta}$ is said to be an $h$ admissible curve if there is a nested sequence of reduced angles at $e^{i \theta}\left\{\alpha\left(\theta, a_{n}, b_{n}, h\right)\right\}$ with $b_{n}-a_{n} \rightarrow 0$, such that each angle contains a truncated subarc $\gamma_{r_{n}}$ of $\gamma$. Let

$$
\pi^{*}(f, \theta, h)=\bigcap_{\gamma} C(f, \theta, h),
$$

where the intersection is over all $h$-admissible curves $\gamma$ at $e^{i \theta}$. If we restrict the reduced angles to be either all right or left reduced $h$-angles in these two definitions we write $R \pi, R \pi^{*}$ or $L \pi, L \pi^{*}$ as the corresponding cluster sets. Suppose $h$ is a convex approach function and $e^{i \theta}$ is both a reduced $h$-Plessner and an $h$-normal point of $f$. It is easy to see that

$$
\pi^{*}(f, \theta, h)=W .
$$

In addition if there is some value $w_{0} \notin R \Lambda(f, \theta, h)$, then we can prove

$$
w_{0} \in R \pi(f, \theta, h) \text {; }
$$

with a similar result holding for left $h$-angles. To show (4.4) consider the reduced (truncated) right $h$-angle in which $f$ omits $w_{0}$. Because $e^{i \theta}$ is a reduced $h$-Plessner point of $f$ we can find a sequence $\left\{z_{n}\right\}$ in a smaller $h$-angle inside of which $f\left(z_{n}\right) \rightarrow w_{0}$ and so there is a $\rho_{0}>0$ such that $f$ omits $w_{0}$ in $N\left(z_{n}, \rho_{0}\right)$ for $n$ sufficiently large. Because $e^{i \theta}$ is an $h$-normal point this implies that in any $\Omega\left(\theta, h^{c}\right)$ containing the reduced right angle $f$ tends to $w_{0}$ in $\bigcup_{n=1} N\left(z_{n}, \rho\right)$, for any choice of $\rho>0$. Each curve tending to $e^{i \theta}$ in any reduced right $h$-angle meets each of the $\operatorname{sets} N\left(z_{n}, \rho\right)$ for some value $\rho$ and $n$ large. Thus $w_{0}$ is in the cluster set of $f$ along all curves in the reduced right angle and (4.4) holds. These remarks together with Theorem 2 give

THEOREM 3. Let $f$ be meromorphic in $D$ and $h$ a convex approach function. Then in a strong sense the reduced h-Plessner points of $f$ are either $h$-Picard points or points $e^{i \theta}$ at which both

(i) $\pi^{*}(f, \theta, h)=W$;

(ii) $R \Lambda(f, \theta, h) \cup R \pi(f, \theta, h)=W=L \Lambda(f, \theta, h) \cup L \pi(f, \theta, h)$.

This theorem strengthens results of Colwell [4, Theorem 3] and Dragosh [7, Theorem 10 (i), (ii), (iii)] in the case $h(\theta)=\theta$; and Yoshida's result [18, Theorem 1], if $h(\theta)=\theta^{q+1}, q \geqq 0$.

We could restate Theorem 3 for a sequence of approach functions but do so only for the polynomial sequence $\mathscr{P}$ for then reduced 
angles and angles are equivalent and a more familiar appearing theorem results.

THEOREM 3 . Let $f$ be meromorphic in $D$. Then in a strong sense the Plessner points of $f$ are either $\mathscr{P}$-Picard points or points $e^{i \theta}$ at which both

(i) $\pi^{*}\left(f, \theta, h_{n}\right)=W$, and

(ii) $R \Lambda\left(f, \theta, h_{n}\right) \cup R \pi\left(f, \theta, h_{n}\right)=W=L \Lambda\left(f, \theta, h_{n}\right) \cup L \pi\left(f, \theta, h_{n}\right)$, for each $h_{n} \in \mathscr{P}$.

We close by showing how our results contain result of Yoshida on pre-Meier points previously referred to. Theorem $1^{\prime}$ tells us that strongly points of $C$ are $K_{h}(f)$ points while Theorem $2^{\prime}$ say that strongly points are either $h$-Picard or $h$-normal point. If we restrict $h(\theta)=\theta^{q+1}, q \geqq 0$, and suppose $e^{i \theta}$ is an $h$-normal point contained in $K_{h}(f)$ but is not an $h$-Plessner point, then for any angle $\alpha(\theta, h)$

$$
\pi^{*}(f, \theta, h)=C(f, \alpha(\theta, h)) \varsubsetneqq W .
$$

A point satisfying (4.5) is called an $h$-pre-Meier point. Of course any $h$-Picard point of $f$ is already an $h$-Plessner point of $f$ so we have shown the result of Yoshida in [19, Theorem 1].

THEOREM 4. Let $f$ be meromorphic in $D$ and $h(\theta)=\theta^{q+1}, q \geqq 0$, then strongly on $C$ points are either h-Plessner points or h-pre-Meier points of $f$.

5. Although we did not use the idea of order of curves, for any approach function $h$ the two boundary curves of reduced $h$-angles have the same order of contact. According to an earlier result of Seidel and Walsh [11, p. 200] the non-Euclidean Frechet distance (and hence the non-Euclidean Hausdorff distance) between the two boundary curves is finite.

I wish to thank Professor Charles Belna for his assistance in the preparation of this paper.

\section{REFERENCES}

1. J. M. Anderson, Boundary properties of meromorphic functions, Quat. J. Math., (2) 8 (1967), 103-107.

2. E. F. Collingwood, On sets of maximum indetermination of analytic functions, Math. Z., 67 (1957), 377-396.

3. E. F. Collingwood and A. J. Lohwater, Theory of Cluster Sets, Camb. Univ. Press, New York, 1966.

4. P. Colwell, Improved versions of forms of Plessner's theorem, Canad. J. Math., (1) XXVI (1974), 251-255.

5. E. P. Doľ̌enko, Boundary properties of arbitrary functions, Izv. Acad. Nauk SSSR, 
31 (1976), 3-14 (Russian); English translation: Math. USSR-Izvestija, 1 (1967), 1-12.

6. S. Dragosh, Horocyclic cluster sets of functions defined in the unit disc, Nagoya Math. J., 35 (1969), 53-82.

7. - The spherical derivative of meromorphic functions, J. Reine Angew. Math., 252 (1972), 51-67.

8. P. Gauthier, A criterion for normalcy, Nagoya Math. J., 32 (1968), 277-282.

9. L. H. Lange, Sur les circles de remplissage non-Euclideans, Ann. Sci. Êcole Norm. Sup., (3) 77 (1960), 257-280.

10. K. Meier, Über die Randwerte der meromorphen Funktionen, Math. Ann., 142 (1961), 328-344.

11. W. Seidel and J. L. Walsh, On the derivative of functions and analytic in the circle and their radii of univalence and of p-valence, Trans. Amer. Math. Soc., 52 (1942), 128-216.

12. U. V. Stayanarayana and M. L. Weiss, The geometry of convex curves tending to 1 in the unit disc, Proc. Amer. Math. Soc., 41 (1973), 159-166.

13. N. Yanagihara, Angular cluster sets and oricyclic cluster sets, Proc. Japan Acad., 45 (1969), 423-428.

14. H. Yoshida, Angular cluster sets and holocyclic angular cluster sets, Proc. Japan Acad., 1 (1971), 120-125.

15. - Tangential boundary properties of arbitrary functions in the unit disc, Nagoya Math. J., 46 (1972), 111-120.

16. - Generalizations of Noshiro's theorem and their applications, Canad. J. Math., XXV (3) (1973), 611-620.

17. - On the boundary properties and the spherical derivatives of meromorphic functions in the unit disc, Math. Z., 132 (1973), 51-68.

18. - On some generalizations of Meier's theorems, Pacific J. Math., (2) 46 (1973), 609-621.

19. Tangential boundary behaviors of meromorphic functions in the unit disc, J. Fac. Eng. Chiba Ann., 25 (1974), 91-98.

20. On the boundary behavior of holomorphic functions in the unit disc, J. Austral. Math. Soc. Ser A, XXI (1975), 36-48.

21. L. Zajiček, Sets of $\sigma$-poposity and sets of $\sigma$-porosity $(q)$, Časopis Pest. Math., 101 (1976), 350-359.

Received October 15, 1977 and in revised form November 3, 1977. This research was conducted while the author was on sabbatical leave from the Pennsylvania State University, U.S.A., and was supported in part by a Canadian Research Council Research grant administered by Carleton University, Ottawa, Ontario.

Pennsylvania State University

UNIVERSITY PARK, PA 16802 



\section{PACIFIC JOURNAL OF MATHEMATICS}

\section{EDITORS}

RICHARD ARENS (Managing Editor)

University of California

Los Angeles, California 90024

C. W. CURTIS

University of Oregon

Eugene, OR 97403

C. C. MOORE

University of California

Berkeley, CA 94720

\section{J. DUGUNDJI}

Department of Mathematics University of Southern California Los Angeles, California 90007

R. Finn aNd J. Milgram Stanford University Stanford, California 94305

\section{ASSOCIATE EDITORS}

E. F. BeCKenbaCH

B. H. NeumanN

F. WOLF

K. YosHIDA

\section{SUPPORTING INSTITUTIONS}

UNIVERSITY OF BRITISH COLUMBIA CALIFORNIA INSTITUTE OF TECHNOLOGY UNIVERSITY OF CALIFORNIA MONTANA STATE UNIVERSITY UNIVERSITY OF NEVADA, RENO NEW MEXICO STATE UNIVERSITY OREGON STATE UNIVERSITY UNIVERSITY OF OREGON
UNIVERSITY OF SOUTHERN CALIFORNIA STANFORD UNIVERSITY UNIVERSITY OF HAWAII UNIVERSITY OF TOKYO UNIVERSITY OF UTAH WASHINGTON STATE UNIVERSITY UNIVERSITY OF WASHINGTON 


\section{Pacific Journal of Mathematics}

\section{Vol. 76, No. $1 \quad$ November, 1978}

Ata Nuri Al-Hussaini, Potential operators and equimeasurability ......... 1

Tim Anderson and Erwin Kleinfeld, Semisimple nil algebras of type $\delta . \ldots .99$

Stephen LaVern Campbell, Linear operators for which $T^{*} T$ and $T+T^{*}$

commute. III ......................................

Robert Jay Daverman, Special approximations to embeddings of codimension one spheres...............................

Donald M. Davis, Connective coverings of $\mathrm{BO}$ and immersions of projective

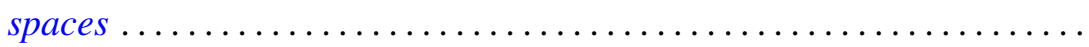

V. L. (Vagn Lundsgaard) Hansen, The homotopy type of the space of maps of

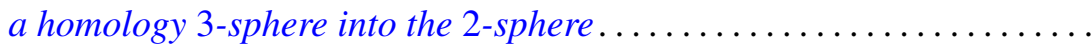

James Victor Herod, A product integral representation for the generalized

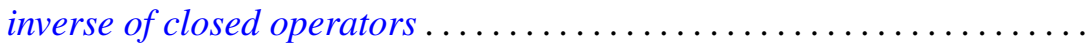

A. A. Iskander, Definability in the lattice of ring varieties ..............

Russell Allan Johnson, Existence of a strong lifting commuting with a

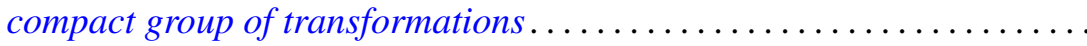

Heikki J. K. Junnila, Neighbornets...................... 83

Klaus Kalb, On the expansion in joint generalized eigenvectors . ......... 109

F. J. Martinelli, Construction of generalized normal numbers . . . . . . . . . 117

Edward O'Neill, On Massey products ....................... 123

Vern Ival Paulsen, Continuous canonical forms for matrices under unitary

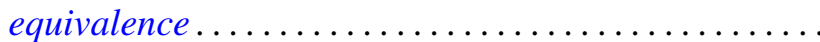

Justin Peters and Terje Sund, Automorphisms of locally compact groups . . . 143

Duane Randall, Tangent frame fields on spin manifolds . . . .

Jeffrey Brian Remmel, Realizing partial orderings by classes of co-simple sets . . . .

J. Hyam Rubinstein, One-sided Heegaard splittings of 3-manifolds ...

Donald Charles Rung, Meier type theorems for general boundary approach

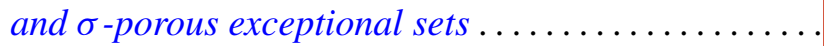

Ryōtarō Satō, Positive operators and the ergodic theorem

Ira H. Shavel, A class of algebraic surfaces of general type constructed from

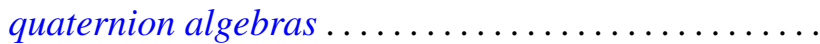

Patrick F. Smith, Decomposing modules into projectives and injectives ....

Sergio Eduardo Zarantonello, The sheaf of outer functions in the polydisc... 